\title{
Choice reaction time with a random foreperiod
}

\author{
DAVID M. GREEN, ALBERT F. SMITH, and SUSANNE M. von GIERKE \\ Harvard University, Cambridge, Massachusetts
}

\begin{abstract}
Choice reaction latencies were measured at three different a priori probabilities for two stimulus alternatives. Unlike the results of some other studies, the mean latency of a given response was nearly the same whether the response was correct or incorrect. The discriminable stimuli were a $1000-$ or a $1700-\mathrm{Hz}$ tone presented at $70 \mathrm{~dB}$ SPL. Latencies and standard deviations, based on about 17,000 observations, are reported for three observers. The data are compared with predictions of the optimum sequential model of Wald and Stone and two modifications of that random-walk model, one proposed by Link and Heath and the other proposed by Laming. Fast-guess analyses were also carried out. The three-parameter version of either the sequential or the modified random-walk models provided reasonably accurate predictions of the mean data for each observer. The parameters estimated by the fast-guess analysis were unrealistic. There are three obvious differences between this experiment and most previous choice reaction-time experiments. First is stimulus modality-we used an auditory signal, whereas most of the previous studies used a visual signal. Second, the observers practiced more in this experiment than in most previous experiments. Finally, there was a random foreperiod with a heavy penalty for anticipations. One or more of these factors is the probable reason for the discrepancy between our results and those of previous studies.
\end{abstract}

Since Wald's (1947) work on sequential testing, and Stone's (1960) suggestion of the relevance of such analysis to reaction-time data, the sequential probability ratio test (SPRT) has been considered a normative, if not descriptive, model of choice reactiontime data. The most startling prediction of Wald's model is that the entire distribution of reaction times for a given response is independent of whether that response is correct or incorrect. Although this very strong prediction was initially supported in the work of Fitts (1966), it has not been found in later studies (Pickett, 1967; Schouten \& Becker, 1967; Wolfendale, 1967). Two recent studies measured thousands of reaction times and found the mean reaction time for correct and incorrect responses to be very different (Laming, 1968; Link, 1975). These investigators suggested two alternative random-walk models.

Link (1975) has abandoned the key assumption of the SPRT model, namely that the decision depends only on the likelihood ratio, and in its place has assumed that the random walks, conditional on either stimulus, are mirror images of each other. Since this assumption can be phrased in terms of

This research was supported in part by the National Science Foundation. The senior author wishes to thank All Souls College and the Experimental Psychology Department of Oxford University for their support during a sabbatical year. We appreciate the comments of R. S. Nickerson and G. Bruce Hennig. To D. R. J. Laming we owe particular thanks. Although we had many divergences of opinion, his comments were very useful and helpful in the process of revising this manuscript. The authors' mailing address is: Laboratory of Psychophysics, Harvard University, Cambridge, Massachusetts 02138. symmetry, about zero, of the random-walk distribution, we call it the SSR (symmetric sampling representation), following a suggestion by Luce (Note 1). This assumption does not force the mean times for correct and incorrect responses to be equal.

Figure 1 summarizes the main assumptions of the SPRT and SSR models and identifies the key parameters. Both models assume a random walk starting at time zero, the onset of the signal. The solid line illustrates the mean drift rates for each model, one given stimulus A, the other given stimulus B. Sampling continues as long as the process is between the two borders, marked A and B. When either boundary is reached, the appropriate response is initiated and, as we will later discuss, the response occurs some time later.

Calculating the distribution of crossing times, indeed, calculating the mean crossing time, is difficult because one must calculate a time to cross the border, given that it has not occurred earlier. The mean time to cross border $A$ then depends on the position of border B, since some sample tracts will cross the "incorrect" border. The formulas for the mean crossing times are complex (see the Appendix). Derivations of these equations can be found in Laming (1968), Link (1975), Link and Heath (1975), Wald (1947), and Luce (Note 1), and are obtained from the momentgenerating function of the sampling process. For the SPRT model, the average drift rate toward the correct border is $\mu_{\mathrm{i}} / \Delta$, where $\mu_{\mathrm{i}}$ is the average step toward the "correct" border and $\Delta$ is the time required for each step. In general, the drift rates are different for the two stimulus alternatives, as illustrated in the 


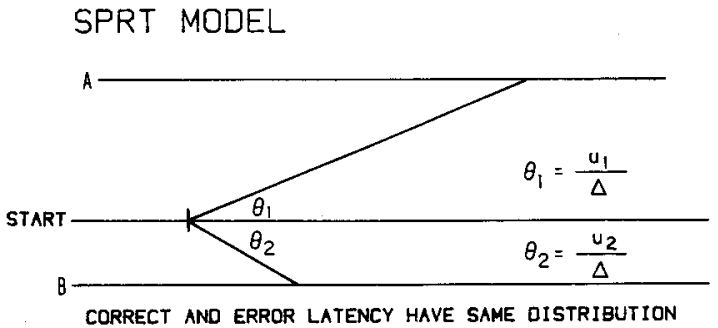

SSR MODEL

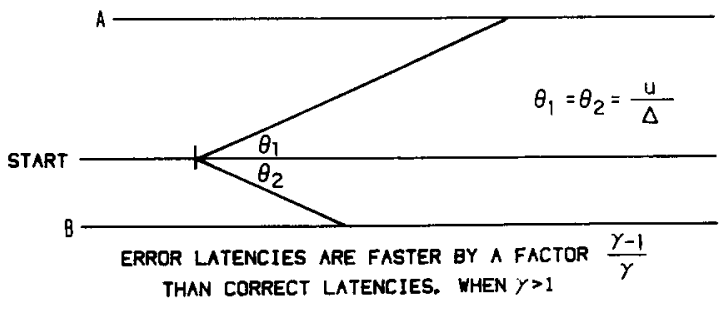

Figure 1. Schematic representation of the random walk and the parameters of the sequential probability ratio model (SPRT) and the symmetric sampling representation (SSR) model. The borders are $A$ and $B$, and once the process crosses those borders response 1 or response 2 is initiated. The parameters $\theta_{1}$ and $\theta_{2}$ represent the average drift rate toward the border. The definition of $\theta$ in terms of the parameters of the model is indicated in the Appendix. These parameters are estimated from the probabilities of the various stimulus response combinations and the associated latencies. Their estimates are given in Table 1.

figure. The surprising result is that, for a given response, the mean time to cross is the same, independent of the stimulus! Indeed, the entire distribution of times to reach either border is dependent only on which border is crossed and is independent of which stimulus is presented.

For the SSR model, the average drift rate toward the correct border is the same for both stimulus alternatives, namely $\mu / \Delta$. As stated previously, the distributions of random walks, conditional on which stimulus is presented, are symmetric about the origin. However, in the general case, each distribution may be asymmetric about its own mean. The degree of asymmetry about the mean is represented by a single parameter $\gamma$. Again the calculation of the time to cross a border is complex. The formulas are given in the Appendix, and derivations of these equations can be found in Link and Heath (1975) and Luce (Note 1). As Link and Heath (1975) derived, the mean difference in time for correct and incorrect latencies, given either stimulus alternative, can be very simply expressed as $(\gamma-1) / \gamma$. The parameter $\gamma$ then characterizes the difference in latencies for correct and incorrect responses; for example, only if $\gamma=1$ are correct and incorrect latencies equal. Generally, errors are faster than correct responses, and thus $y$ is assumed to be greater than unity.

Laming suggests that the failure of the randomwalk models occurs because the observer may begin to sample, at least on some trials, before the stimulus alternative is presented. These presamples will lead to some random displacement of the starting point and to response latencies that depend on whether the response is correct or incorrect. Laming (1968, 1979b) further supports this presampling view with data on the autocorrelation of successive response times. Positive autocorrelations over the course of a few trials can be interpreted to reflect slow drift, in the onset of the presampling interval, over successive trials. Laming does not present a theory of sufficient quantitative detail to allow us to predict the observed mean latencies. Rather, his views are essentially excuses (albeit supported by detailed analyses of his data) for the failure of the existing random-walk models and suggestions as to important variables neglected by existing theories. Since the amount and extent of presampling is under the observer's control, either we must develop ways to estimate it and incorporate it into theories predicting the observed reactiontime latencies or we must devise procedures to severely discourage such behavior. Another tack would be to encourage presampling by lowering the penalty for anticipations. The amount of presampling may also be related to the modalities in which the warning signal and reaction signal are presented. In most previous experiments, the warning signal is visual, as is the reaction signal. In this experiment, we used a visual warning signal and an auditory reaction signal. Perhaps presenting the two signals to different modalities discourages presampling.

A troublesome fact, at least to us, is that previous data are based entirely on averages over a number of observers, with each observer contributing only several hundred observations. Thus, the SPRT model may fail simply because none of the observers has had sufficient practice to reach asymptotic performance levels. In addition, both Laming's and Link's experiments used a fixed interval between the ready signal and the occurrence of one of the two potential stimuli. This procedure can easily lead to faster reaction times when wrong than when right, simply because the observer is trying to achieve speed at the expense of accuracy and therefore occasionally initiates anticipations or "fast-guess" responses.

There are several major differences between the procedures used in this study and those employed in previous studies. First, we measured choice reaction times over an extended period of time for three highly trained observers. We presented data and the theoretical analyses for each observer separately. Second, in an effort to discourage presampling or fast-guess behavior, we imposed a random period 
of time between the beginning of a trial and the onset of the reaction signal (random foreperiod). A severe penalty was imposed on any response given before the signal onset. Finally, we employed auditory stimuli rather than visual stimuli, which were commonly used in the previous studies. In our opinion, the single most important change was the introduction of the random foreperiod. This change was intended to minimize the occurrence of non-stimulus-related responses and to achieve an experimental situation more consonant with the theoretical analyses.

Evidence of the effectiveness of one or more of these differences in procedure may be seen in Figure 2 , which shows the mean reaction time for correct and incorrect responses. The two means, averaged over the three observers, are plotted for the three values of a priori probability used in this experiment. Similar mean latencies, averaged over 24 observers in Laming's experiment and over $4 \mathrm{ob}$ servers in Link's experiment, are also plotted in Figure 2. Two things are clear. First, our auditory discrimination is apparently made more quickly than the visual discriminations of the other experiments. Second, in our data there is considerably less tendency for the correct response to be slower than the incorrect response.

In the following sections we explain our procedure in detail, present our results, and compare the data with predictions of the SSR, the SPRT, and the fastguess models.

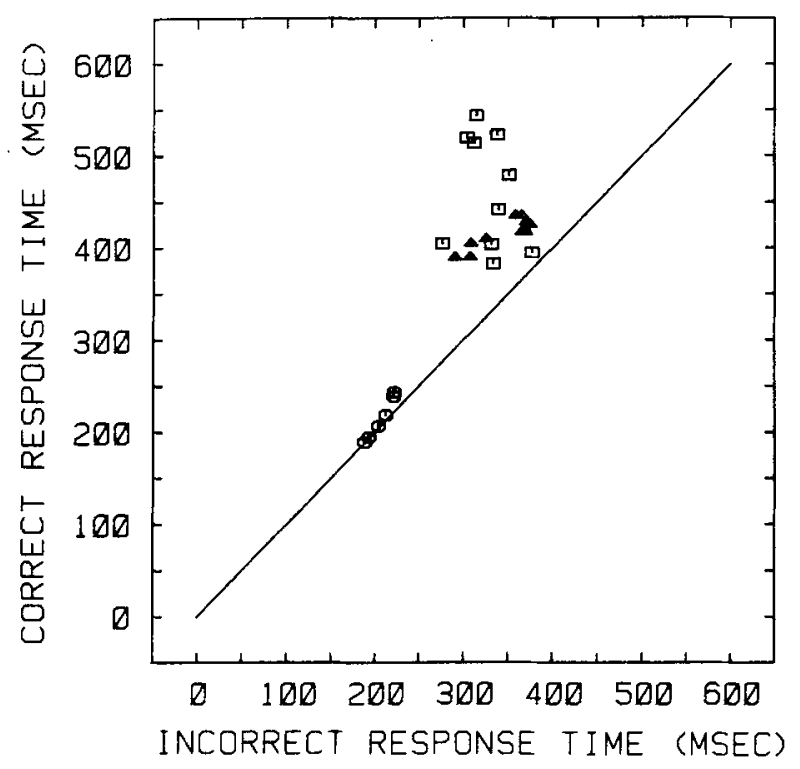

Figure 2. The average latencies for a correct or incorrect response in severnal choice reaction-time experiments. The avernge, over subjects, is computed for each a priori condition studied in the different experiments. Data from the present experiment is shown as circular points (two responses and three conditions), Link's data are the squares (two responses and five conditions), and Laming's data are indicated by the triangles (two responses and five conditions).

\section{METHOD}

For the observer, a trial begins with a countdown of five 100 msec events presented on a television terminal. At the end of the last event, a random wait with density $\mathrm{e}^{-t}$ occurred, where $t>0$ is measured in seconds. The mean wait is $1 \mathrm{sec}$ and the variance is also $1 \mathrm{sec}$, but the modal wait is zero. At the end of the wait, a 1000 - or $1700-\mathrm{Hz}$ tone was presented at $70 \mathrm{~dB}$ SPL. For these three observers, the level was about $70 \mathrm{~dB}, 3 \mathrm{~dB}$ above their absolute thresholds. Thus, the signal levels are also approximately $70 \mathrm{~dB}$ SL. The tone was turned on abruptly, starting at a zero crossing of the sinusoid. The observers, who listened binaurally over TDH-50 earphones in sound-treated rooms, were instructed to push one of two buttons, depending on the frequency of the signal. The buttons were about $3 \mathrm{~cm}$ in diameter (Refac Model TC-1/M7) and were located side by side, $3.2 \mathrm{~cm}$ between centers. A very small travel (approximately $0.01 \mathrm{~cm}$ ) is needed to set these switches.

A system of points was associated with the various possible events that could occur on any trial. Points were given to responses that occurred within $380 \mathrm{msec}$ following the signal onset, according to a linearly decreasing function starting with 5 points at zero time and diminishing to no points at $380 \mathrm{msec}$. Responses occurring more than $380 \mathrm{msec}$ after signal onset were penalized 2 points. An incorrect response (but one occurring after signal onset) was penalized 5 points independent of its latency. If a response occurred before the signal was presented, what we call an anticipation, a penalty of $\mathbf{5 0}$ points was imposed. Because of the severe penalty for anticipations, they occurred rarely; 9 anticipations occurred in the more than 50,000 responses. After each response, a cumulative total of the points obtained in the run was flashed on the screen for $750 \mathrm{msec}$, followed by a $500-\mathrm{msec}$ wait, and then the countdown for the next trial began.

The three observers, all in their early 20 s, had had different amounts of practice in reaction-time experiments. One, D.S., had participated for $S$ weeks in a simple reaction-time experiment and had recorded over 15,000 responses. Another, S.V.G., had practiced for a little over a week immediately before the experiment and had made about 5,000 responses in a choice reaction experiment. The third observer, C.E., was given 3 days of practice before this experiment began-about 3,600 responses.

Three levels of a priori probability for a particular stimulus were tested: $25 \%, 50 \%$, and $75 \%$. Only one a priori level was used on any given day. Sessions of 100 trials were run, lasting about $5 \mathrm{~min}$. A typical day consisted of running 12 runs of 100 trials, or 1,200 reactions. Blocks consisted of 3 days of observation with all three a priori levels represented. The order of a priori levels was always run in the order $50 \%$, then $25 \%$, and then $75 \%$ within blocks. Observer D.S. produced six anticipations, three each in the $25 \%$ and $75 \%$ conditions. Observer C.E. produced two anticipations, one each in the $25 \%$ and $50 \%$ conditions. $\mathrm{Ob}$ server S.V.G. produced a single anticipation in the $75 \%$ condition.

\section{RESULTS}

\section{Practice Effects}

Because of the random foreperiod, all reaction times are measured from the onset of the signal, not the beginning of a trial. Despite the considerable experience of the observers, we noted a gradual decrease in the mean reaction time as the experiment progressed. Figure 3 shows the weighted mean reaction time, averaged over right and wrong responses, as a function of block number-that is, averages over the 3 successive days representing all three levels of a priori signal probability. Each mean is based on about 3,600 responses, and a small but noticeable trend is evident. Because of this trend, we report 


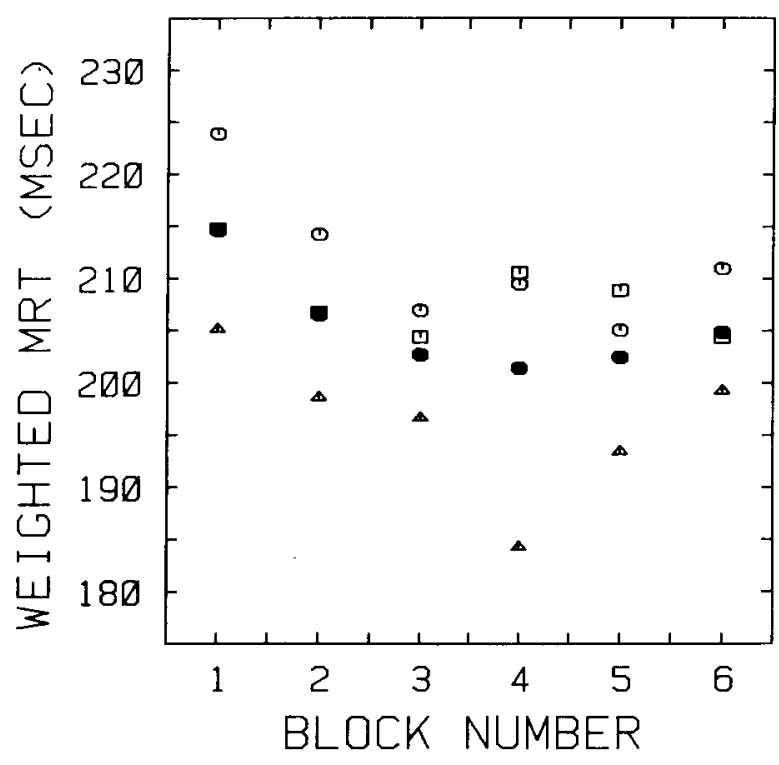

Figure 3. The weighted mean reaction time computed over successive 3-day sessions (blocks). Each block represents all three a priori probabllity conditions and represents about 3,600 observations, about 1,200/day. The obiervers are coded as follows: D.S., circles; C.E., triangles; and S.V.G., squares.

data from only the last five blocks, approximately the last 17,500 responses for each observer, where the means, at least on average, are reasonably stable and no clear practice effects are evident.

\section{Mean Latencies}

The mean latencies for each stimulus-response combination for three a priori probabilities and for the three observers are shown in Table 1. In the top portion of the table are the averaged data. For each a priori condition, the four possible stimulus-response conditions are listed. The mean latency, in milliseconds, standard deviation, and number of responses are recorded for each condition. At the bottom of the table are the parameters of the two major theoretical models, SPRT and SSR. The values A and $B$ are the boundaries of the random-walk process and are the same for each model (see the Appendix or Figure 1). For both models, a three-parameter fit was obtained, $\Delta / \mu_{1}, \Delta / \mu_{2}$, and $\mathrm{r}$ for the SPRT model or $\Delta / \mu, \gamma$, and $\mathbf{r}$ for the SSR model. For both models, an additional parameter representing a different delay for each response was also estimated, yielding the four-parameter model. Basically, the parameters were obtained by estimating the linear regression parameters in a three- or four-variable equation. The dependent variables of the regression are the mean reaction times, that is, four latencies at each of the three levels of a priori probability.

This method of estimating parameters is not the most desirable. The problem is that the regression variables $\eta_{\mathrm{ij}}$ are functions of the correct and error probabilities and hence subject to error. This situation can produce biased estimators (see Kendall \& Stuart, 1960, Vol. 2, chap. 29). We ran a simulation in which the steps of the random walk came from two equal-variance gaussian distributions whose means differed in sign. This satisfies both the SSR and SPRT models. Using about the same number of simulations as we ran trials in the experiment, we used the same procedure for estimating parameters from the output of the simulations. About 20 to 50 steps occurred in the simulations before a border was reached. Thus, there was little excess over the boundary (see Wald, 1947). No bias was evident for any of the estimates. The boundary values $A$ and $B$ were estimated to within $5 \%$ at worst, and other estimates were even better. Although we would like to estimate the parameters in some other way, we know of no practical alternative.

Since the SPRT model predicts that the mean latency for a given response is independent of the stimulus, we computed a weighted mean latency for each response and used that quantity as the dependent variable of the regression equation.

The independent variables of the linear equation are the residual latency (r) and two quantities $\eta_{\mathrm{ij}}$, weighting the theoretical parameters $\Delta / \mu_{1}$ and $\Delta / \mu_{2}$ for the SPRT model and $\Delta / \mu$ for the SSR model. The $\eta_{\mathrm{ij}}$ parameters are functions of $\mathbf{P}_{\mathrm{ij}}$. (See the Appendix for exact equations for each model.) The fourth parameter was the response used by the observer, either +1 for response 1 or -1 for response 2. Twice this quantity is the presumed difference in latency needed to execute the different responses.

The values of all the parameters are in a reasonable range. The estimated residual latency is as small as $146 \mathrm{msec}$ (for Observer C.E.) and as large as 173 msec (for Observer D.S.). For the SPRT models, the values of $\Delta / \mu_{1}$ and $\Delta / \mu_{2}$ are opposite in sign and roughly equal in size, as one might expect. The values of these parameters change appreciably, however, if four rather than three parameters are estimated. For example, the value of $\Delta / \mu_{2}$ changes about $50 \%$ for D.S. and C.E. The value of $y$ in the SSR model reflects the asymmetry in the random walk around its mean and is near, but not equal to, unity. The parameters of this model are not greatly altered when four rather than three parameters are estimated. This lack of change is consistent with the result that the differential weight given to one or the other response is very small, no greater than $6 \mathrm{msec}$. The SPRT models suggest that the difference in mean latency to accomplish the two responses is sizable $(2 \times 18.8$, or $37 \mathrm{msec}$ ) for D.S. We will discuss this topic further when we present data on the latency of a simple reaction time for each response. Once the regression estimates are complete, we have a predicted and obtained mean latency and can compute the RMS dif- 
Table 1

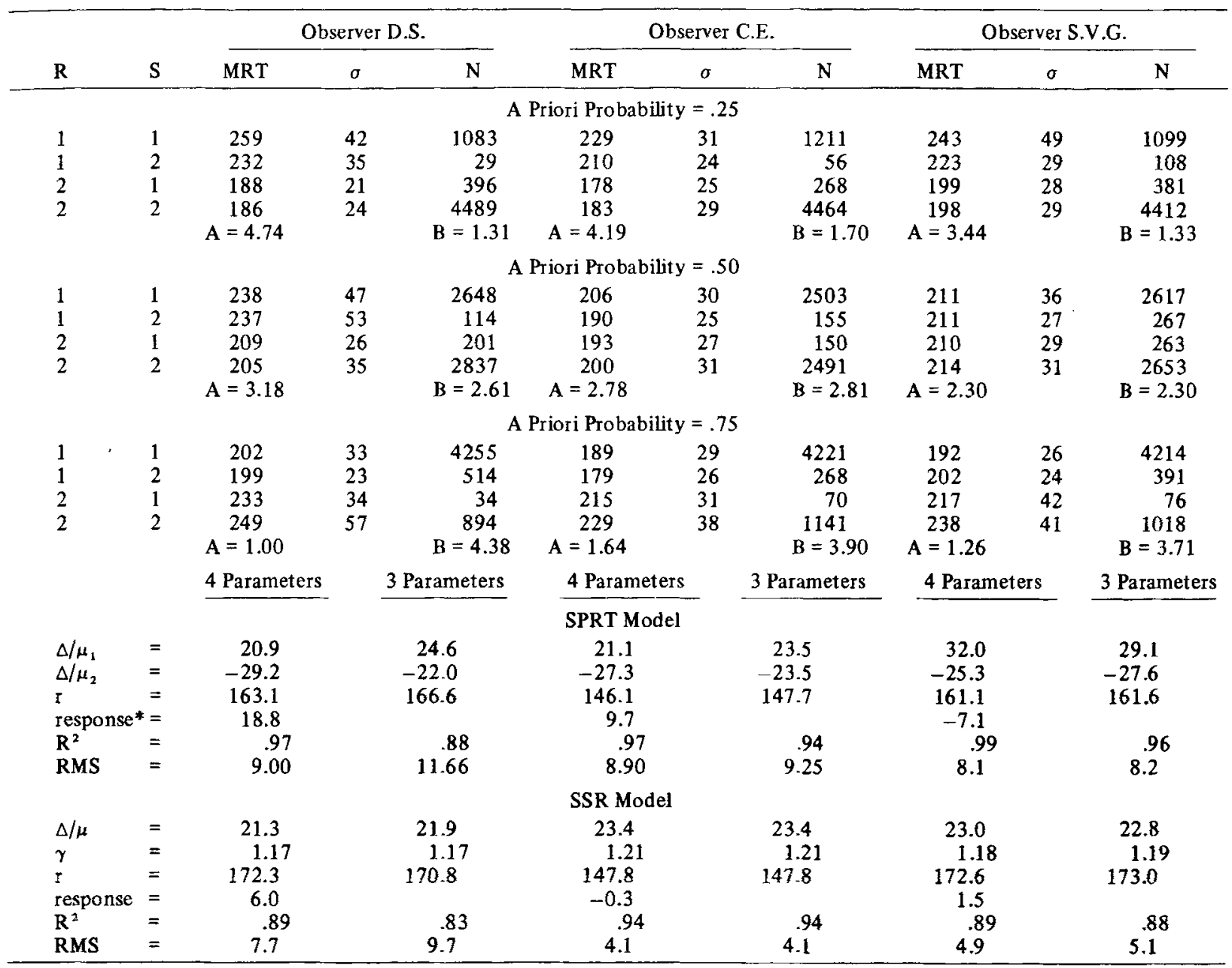

Note-MRT and o given in milliseconds. $\quad$ *Left response, +1 ; right response, -1 .

ference between them. These values are listed in the table.

For the SPRT model, the weighted average of right and wrong responses is used as the dependent variable in the multiple regression equation. The average mean deviation from this weighted mean is therefore zero in the regression. In our computation of the RMS error for this model, we have used each response, whether right or wrong. Our mean error is therefore close but not exactly equal to zero, and the RMS error is about $10 \%$ higher than what we could obtain by forcing the mean error to zero. For the SSR model, this problem does not arise, because all 12 mean latencies are used in the original multiple regression equation.

We also include the Pearson product moment $\left(R^{2}\right)$ between the obtained mean latencies and means predicted by the multiple regression equation. Neither the difference in correlation nor the size of the RMS error is sufficiently different to discriminate between the two models. The largest RMS error is about $10 \mathrm{msec}$, and the standard deviation of a typical latency is about $20-40 \mathrm{msec}$, so the two models predict the mean latencies with reasonable, and roughly equal, accuracy.

\section{Response Latency as a Function of Foreperiod Wait}

The use of a random foreperiod means that the stimulus will be presented at different intervals of time following the warning signal. One may well wonder if the different wait intervals affect the reaction-time latency. Note that this analysis is probably somewhat different from many previous studies of the effect of foreperiod interval. When the inter$\mathrm{val}$ is fixed, or even randomized over a few discrete values, the subject can occasionally time-estimate the interval and initiate fast-guess responses, that is, responses unrelated to the stimuli that are presented on those trials (Ollman, 1966; Snodgrass, 
1969; Snodgrass, Luce, \& Galanter, 1967; Yellott, 1967). Nickerson (1967) and Nickerson and Burnham (1969) have most thoroughly investigated the truly random or "nonaging" foreperiod for simple reaction times. In the truly random foreperiod, the probability that the stimulus is going to appear in the next small interval of time is fixed and constant, and independent of the amount of time that has elapsed.

To analyze how the foreperiod interval affects the latency of response, we sorted the responses into categories corresponding to the foreperiod wait. The foreperiod wait, which is exponentially distributed, was divided into 256 intervals such that an equal number of stimuli should occur for each wait. We also separated the correct responses from the error responses, since the equality of the two response latencies is crucial for the SPRT model. Mean response latency was then computed for each of the 256 wait intervals; the smoothed, or running, average of these means is presented in Figure 4.

The abscissa is simply the number of the foreperiod wait where time has been transformed so that an approximately equal number of responses occur at each time. The actual value of time is shown along the bottom of the figure. At the top, we indicate the

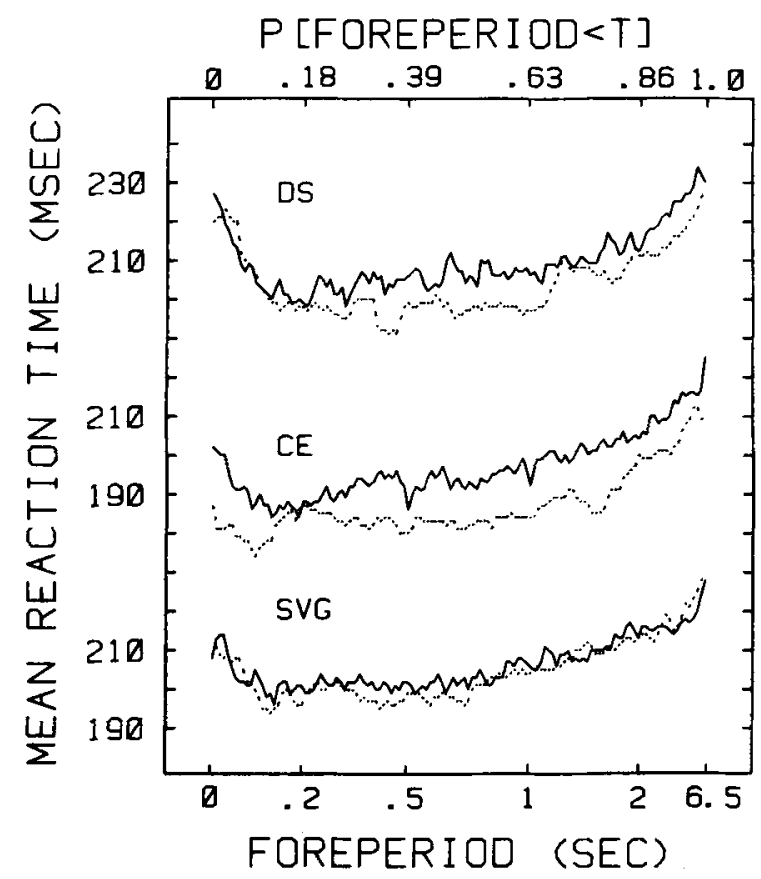

Figure 4. Mean latency of response for correct (solid line) or incorrect (dotted line) as a function of the foreperiod wait. The lines represent running averages computed over several wait intervals. The $\mathbf{2 5 6}$ wait intervals were selected so that approximately equal numbers of observations fall into each interval. The time in seconds associated with the intervals is indicated along the bottom abscissa. The probabillty that the foreperiod wait will be equal or less than that time is given along the top abscissa. The point plotted is based on an average over nine intervals for the correct responses and over 31 intervals for the incorrect responses. probability of a foreperiod wait of this amount or less. Thus, above the 1 -sec mark, we find that $63 \%$ of the waits should be less than this value. Similarly, by $3 \mathrm{sec}, 95 \%$ of the waits should have expired.

The ordinate is the smoothed, or average, response latency for that foreperiod interval. A simple cosine window was used to smooth the data. Since there are about 10 correct responses for every error, the smoothing window for the errors was made about $3 \approx 10^{1 / 2}$ times larger so that the variability of the correct and incorrect latencies would be roughly the same. Since each subject showed much the same pattern for the various a priori probability conditions, and because each subject was slightly different from each other, we have presented average data for each subject. Despite these differences, there are some similarities. First, each subject has somewhat greater latencies for the very short foreperiods. If the signal arrives in the first $100 \mathrm{msec}$, as about $10 \%$ of the signals do, then the response is 5 to $20 \mathrm{msec}$ slower than if it occurs somewhat later. Once this initial period is past, the response latency increases very slowly, about 5 to $10 \mathrm{msec}$, depending on the subject, until a wait of 2 to $3 \mathrm{sec}$ has elapsed. By this time, the stimulus will have occurred on $75 \%$ to $95 \%$ of the trials. If a longer wait does occur, the response latency will be increased by 10 to $20 \mathrm{msec}$, depending on the subject.

Nickerson's (1967) data for simple reactions shows a similar trend. In one of his conditions, the mean wait was $1 \mathrm{sec}$, as it was in our data. Nickerson divided his data according to the duration of the wait. He used 10 waits of equal durations, and 0 to $400 \mathrm{msec}$ as the first category. For that wait, the reaction time was somewhat slower (about $10 \mathrm{msec}$ ). After the first $400 \mathrm{msec}$ of wait, the reaction time was largely independent of the wait. About $33 \%$ of the stimuli will occur in that first wait interval, so that the total effect on the overall mean is about $3 \%$.

In the data of the present experiment, these nonhomogeneous effects are also relatively small. A change of $20 \mathrm{msec}$ in 200 is only $10 \%$. Furthermore, of the majority of the trials, encompassing the wait intervals of 0.1 to $1.5 \mathrm{sec}$, the change in response latency is less than $5 \%$. Since the remaining trials represent only $30 \%$ of the data, and since the change is at most $10 \%$, the effect on the overall mean is less than $3 \%$.

What the latency analysis does show is that for two of the three subjects there does seem to be a consistent tendency for the errors to be somewhat faster than the correct responses. Computing the average latency over all foreperiod intervals partially obscures this difference. The difference depends on the subject, being largest for C.E., approximately $10 \mathrm{msec}$, and essentially zero for S.V.G.

A related issue is whether the occurrence of an error is related to the duration of the foreperiod wait. 
To study this issue, we divided the foreperiod wait into 16 categories so that if the errors were independent of wait interval we would expect to find an equal number of errors in each category. We then could compute a simple chi-square test, using 1/16th of the total number of errors as the expected number in each category. The expected chi-square value is 15 and the standard deviation is about 4 . The average chi-square over all observers and conditions is 15.5. The largest chi-square value is 24 , which does not quite reach the $5 \%$ significance level. Thus, the errors seem to occur equally often at all foreperiod intervals.

\section{FURTHER TESTS OF THE MODELS}

\section{The SPRT Model}

According to the SPRT model, not only should the mean latency for a given response be independent of whether it is right or wrong, but also the entire distribution of latencies should be the same. The most straightforward test of the equality of two distributions is the Kolmogorov-Smirnov maximum deviation test (Bradley, 1968, pp. 296-303). Of the 18 such tests, 2 responses, 3 levels of a priori probability, and 3 observers, 14 were significant at the $1 \%$ level and many would be significant at even higher levels of significance. Thus, we may safely conclude that the distributions differ. To explore how the two distributions differed we constructed cumulative distributions of the right and wrong latencies for each response. Each distribution represented the probability that a response latency occurs before some time, $t$. Thus, we can plot one cumulative distribution against the other as the time, $t$, is varied in the manner of an ROC curve. We adopted the convention of plotting the cumulative error distribution along the ordinate and the cumulative correct distribution along the abscissa. If the distributions are the same, the cumulative line should fall along the major diagonal. If one distribution is slower or faster than the other, the line will deviate from the major diagonal. Inspection of the curves showed that most hovered around the diagonal, but that some of the lines lie consistently above or below the diagonal. Thus, a simple and sensitive test of whether or not the distributions are the same is whether the area under the ROC curve is equal to 0.5 . The computed area is shown in Table 2. As can be seen, the area fluctuates about the value of 0.5 and the average value is 0.56 , which means that the error distribution rises somewhat faster than the correct distribution. The area statistic also has very simple statistical properties. Bamber (1975) has described this kind of analysis and points out that this area is proportional to Mann-Whitney's U statistic. For a large number of observations, the variance of the area is gaussianly distributed and has a variance equal to
Table 2

Area Under Cumulative Distributions of Incorrect $(y)$ Versus Correct (x) Latencies

\begin{tabular}{|c|c|c|c|c|c|c|}
\hline \multirow[b]{2}{*}{$\mathbf{R}$} & \multicolumn{2}{|c|}{ Observer D.S. } & \multicolumn{2}{|c|}{ Observer C.E. } & \multicolumn{2}{|c|}{ Observer S.V.G. } \\
\hline & A & $\mathbf{z}$ & $\mathbf{A}$ & $\mathbf{z}$ & $\mathbf{A}$ & z \\
\hline \multicolumn{7}{|c|}{ A Priori Condition .25} \\
\hline $\begin{array}{l}1 \\
2\end{array}$ & $\begin{array}{l}.72 \\
.44\end{array}$ & $\begin{array}{r}2.73 \\
-2.36\end{array}$ & $\begin{array}{l}.69 \\
.55\end{array}$ & $\begin{array}{l}3.15 \\
1.82\end{array}$ & $\begin{array}{l}.63 \\
.49\end{array}$ & $\begin{array}{r}2.82 \\
-.24\end{array}$ \\
\hline \multicolumn{7}{|c|}{ A Priori Condition .50} \\
\hline $\begin{array}{l}1 \\
2\end{array}$ & $\begin{array}{l}.52 \\
.43\end{array}$ & $\begin{array}{r}.49 \\
-1.88\end{array}$ & $\begin{array}{l}.68 \\
.56\end{array}$ & $\begin{array}{l}5.49 \\
1.57\end{array}$ & $\begin{array}{l}.48 \\
.55\end{array}$ & $\begin{array}{r}-.62 \\
1.66\end{array}$ \\
\hline \multicolumn{7}{|c|}{ A Priori Condition .75} \\
\hline $\begin{array}{l}1 \\
2\end{array}$ & $\begin{array}{l}.50 \\
.58\end{array}$ & $\begin{array}{r}-.00 \\
.93\end{array}$ & $\begin{array}{l}.62 \\
.60\end{array}$ & $\begin{array}{l}4.04 \\
1.79\end{array}$ & $\begin{array}{l}.38 \\
.70\end{array}$ & $\begin{array}{r}-5.08 \\
3.71\end{array}$ \\
\hline
\end{tabular}

Note-Mean $A=0.56 ;$ mean $z=1.11 ; \quad R=$ response

$$
\text { Variance }(\text { area })=\text { Area }(1-\text { Area }) / N \text {, }
$$

where $\mathbf{N}$ is the lesser number of latencies in the two distributions. Thus, for each area value we can compute a $\mathrm{z}$ score to indicate how many sigma units the observed area is from 0.5. A value of 1.96 is significant at the $5 \%$ level and 2.60 is significant at the $1 \%$ level. Thus, many values are significant. The average $\mathrm{z}$ score value is 1.11 , indicating, as does the average area, that correct responses are slightly slower than error responses.

The preceding analysis is a good test to see if two distributions are different in location. The area statistic can be 0.5 , however, and the two distributions could be different in variance but have exactly the same mean. The ROC curve for such a case would be an S-shaped curve that crosses the diagonal twice. We tested the two distributions with a simple $F$ test to check the equality of the variance. The majority of tests were nonsignificant, but three conditions showed highly significant $F$ ratios. They are: S.V.G. for response 1 in the $25 \%$ and $50 \%$ conditions and D.S. for response 1 in the $75 \%$ condition. The sigmas for these conditions differ by $9 \mathrm{msec}$ or more (see Table 1). In all three cases, the variance of the correct distribution is larger than the error distribution. Inspection of the ROC curves for these cases showed the expected $S$ curve in two cases and a curve that wandered about the diagonal in the third case. Thus, it appears that there are genuine differences in the variances in some cases.

In summary, the prediction of equal distribution of latencies for correct and incorrect responses can be rejected with some statistical confidence. The difference arises largely because errors are faster than correct response, but the size of this departure is not very great. In some cases, the difference arises because of differences in the variability of the two distributions. 


\section{The SSR Model}

A strong prediction of the SSR model is that a proportional relation should be obtained between the border parameters $A$ or $B$ and the difference between the mean correct and mean incorrect responses. This prediction is easily derived if we subtract A10 from A9 or A11 from A12 (see Appendix). The difference in mean is a linear function of the parameter $\mathbf{A}$ or B with zero intercept. Figure 5 shows plots of this relationship for the three observers and for the data, where we have averaged over the three observers. Except for Observer C.E., the intercept is far from zero, and the intercept for the average is also far from zero. Thus, although the theory can predict the mean responses with some accuracy, this correspondence appears to be fortuitous.

\section{Autocorrelation of Latencies}

As stated previously, Laming has advanced the hypothesis that failure of the SPRT model may arise because the observer samples before any signal is presented. Since these presamples are unrelated to the correct alternative, they lead to asymmetries in the correct and incorrect responses. Laming has also shown that choice reaction-time data shows sizable autocorrelations, which could arise from drifts, over time, in the amount of presampling. We analyzed the present data to determine if such autocorrelations

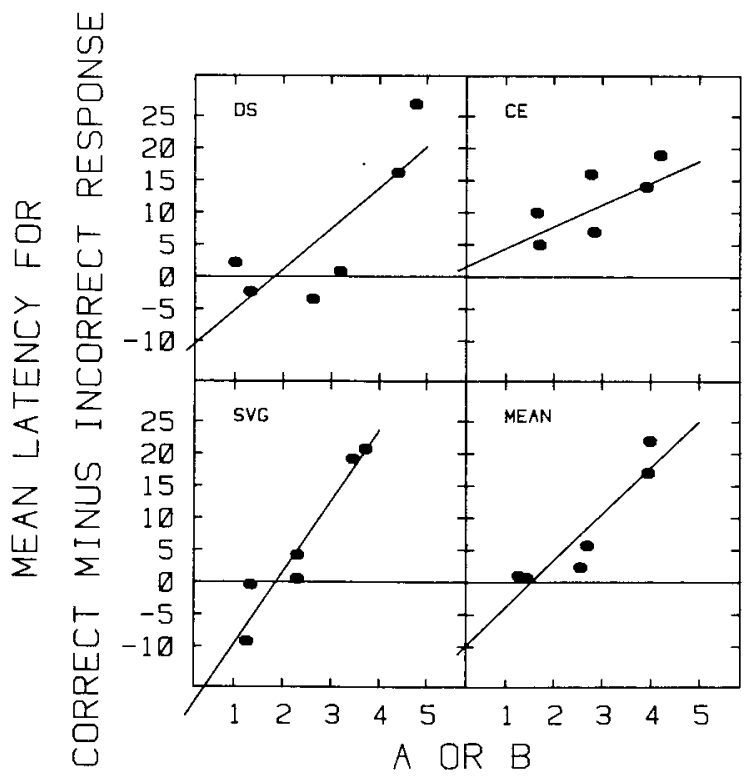

Figure 5. A test of the linear prediction of the SSR model that the mean difference in latency for correct and incorrect response is simply proportional to the boundary value associated with that response, either $A$ or $B$. Three observers are shown with two points for each a priori condition. We have also averaged the data over observers to compare with previous tests of this prediction. The intercept, which should be zero, is not, except perhaps for $\mathrm{Ob}$ server C.E.

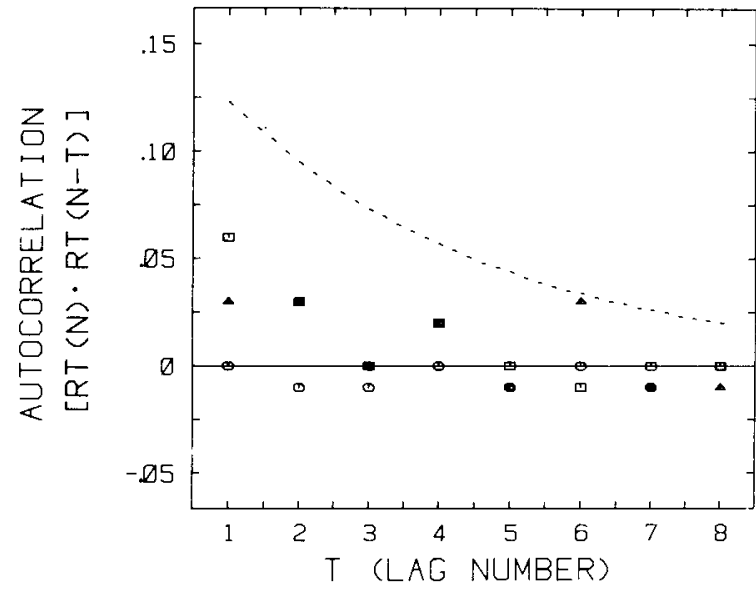

Figure 6. The correlation of the latency on the present trial with the latency $\mathbf{T}$ trials past. The dotted line represents the average of Laming's (1979b, Flgure 1) results. The three observers in this experiment are coded in the following manner: D.S., circles; C.E., triangles; S.V.G., squares.

were apparent. Figure 6 shows a graph of the data. The autocorrelations were computed for each a priori condition separately, and the correlations averaged. None of our observers displayed much of an autocorrelation, even for a lag of one trial. Observer D.S. has no correlation that exceeds \pm 0.01 for any lag or any a priori condition. The other two observers show trends similar to that measured by Laming, but the magnitude of the effect is much smaller. Although we cannot be certain about the causes of the different results, likely candidates are the difference in practice between our observers and his, and/or the difference in modality, and/or the fact that we used a random foreperiod and a heavy penalty for anticipation. The latter should particularly discourage presampling.

We do not believe that the difference lies in the different procedures used to compute the autocorrelation values. In our computation, we simply took the raw latencies, delayed them by the appropriate amount, and computed a standard correlation coefficient. In the appendix to Laming's (1979b) article, is an explanation of an involved computation to estimate the correlation coefficients for various lags. That method, on inspection, is identical, except for round-off error, to our simple, direct estimates. The point Laming is trying to make is that direct estimates can be contaminated by other factors that will reduce or mask the coefficients estimated by the direct approach. The argument for this position and formula for estimating the autocorrelation coefficients can be found in Laming (1968).

The effects of masking, or screening, as it is sometimes called, by some third variable is a well-known topic in regression analysis. Hays $(1963$, p. 575$)$ and 
Kendall and Stuart (1960, p. 331) give the following formula for the case of three variables:

$$
R_{12.3}=\frac{r_{12}-r_{13} r_{23}}{\left[\left(1-r_{13}^{2}\right)\left(1-r_{23}^{2}\right)\right]^{1 / 2}} .
$$

Since the denominator is positive, the increase in the correlation will occur only if $r_{13}$ and $r_{23}$ are opposite in sign. Suppose they are equal and opposite in sign; then some algebra shows that

$$
r_{13}{ }^{2}=r_{23}{ }^{2}=\frac{R_{12.3}-r_{12}}{1-R_{12.3}}
$$

Thus, for example, if the direct correlation $r_{12}$ is 0.05 and the partial $R_{12.3}$ is 0.15 , then the correlation with some other factor must be as large as 0.34 . Furthermore, this correlation with some third factor must be positive for one lag and negative for the other, a most unusual circumstance. One would need to find a variable showing considerably more influence than any found so far in order to use masking as a means of explaining the discrepancy between our estimates of the autocorrelation function and his estimates.

\section{Laming Error Analysis}

Another characteristic of choice reaction behavior observed by Laming is the decrease in the probability of an error following an error on the previous trial. Such a result is intuitively reasonable-one has just made an error and should be more cautious. According to Laming's theory, such behavior is consistent with a sharp decrease in the amount of presampling following an error trial. For example, the proportion of errors dropped from about $6 \%$ to $1 \%$ in one experiment and from $3 \%$ to essentially zero in two other experiments on the trials following an error (Laming, 1979b).

In the data obtained in this experiment, however, the error proportion changed very little. The ratio of the error proportion following an error to that following a correct response was 1.21 for D.S., 1.26 for C.E., and 0.84 for S.V.G. In Laming's data, this ratio was at 0.18 or smaller. Thus, the performance following an error was quite different in the two experiments.

We also computed a $z$ score for the difference in percentage of errors following a correct and incorrect response. The average of these $z$ scores for the three a priori conditions and the three observers was 0.34 . Three of the $z$ scores exceeded 2.0, and thus is "statistically significant," but two of the three were in the direction of an increase in the error percentage following an error trial.

Given the relatively few number of trials used in Laming's experiment, it does not seem surprising that the observer tends to be cautious following an error and to make relatively fewer errors on the subsequent trial. One might also expect that the decrease in error proportion should be accompanied by a slightly longer latency, as Laming observed. The change is about $70 \mathrm{msec}$ in his data. In our data, the observers are more practiced and have participated in the experiment for a much longer period of time. Errors do occur, about 1 in every 10 or 20 responses, but they do not seem to change the observer's behavior on the next trial. The nonhomogeneity in our data is consistent with a general waxing and waning of performance. Periods of better or worse performance, extending over several trials, will tend to make errors come in bunches rather than be evenly distributed throughout the trials. But even this tendency is slight. The change in latency of responses following a correct or incorrect response was also small. There was an overall tendency to go more slowly following an error, but the change in the average latency of any response, correct or incorrect, was only $5 \mathrm{msec}$ when averaged over all conditions and observers.

These differences between Laming's results and ours suggests that either presampling has been eliminated in our experiment or that the pattern of presampling has been altered. Our exponential foreperiod would discourage presampling because such behavior would necessarily lead to occasional anticipations that were heavily penalized. Of course, nine anticipations did occur in the entire experiment, so some presampling may have occurred. The effects observed by Laming can be explained by systematic changes in presamplings as a result of the outcomes on a given trial. Such systematic changes clearly have been altered or eliminated in our experiment.

\section{Simple Reaction-Time Latency}

Both of the models assume a residual component (labeled $r$ in Table 1) that is a random variable and independent of a decision process. In effect, this parameter introduces an additional random delay between the response and the time taken to decide between the two stimulus alternatives. One should note that any delay, as long as it is independent of the choice process, may contribute to this residual. A peripheral sensory delay in processing the signals, a decoding delay, or, most certainly, a motor component involved in executing the response could be part of this residual delay.

Furthermore, in the four-parameter estimation procedure, both models, but particularly the SPRT model, suggested that the delay associated with one response was considerably greater than that associated with the other response. This difference could arise if there were a greater delay in processing one of the two signal frequencies or if one response could be executed more quickly than the other. 
To investigate these matters more fully, we conducted some simple reaction-time tests with our observers. The experimental situation was exactly the same as that used in the choice reaction experiment, except that, by setting the probability of one of the signals to one and the other to zero for a number of trials, we eliminated any uncertainty about which signal would be presented. Thus, in any session only one signal occurred and the observers used only one response. They tried to respond as fast as possible, in order to maximize the number of points. The point schedule was the same as that used in the choice reaction experiment, including the penalty for anticipation.

They were instructed to execute the response in the same fashion as they did in the choice experiment. Two of the observers, C.E. and S.V.G., used the index fingers of each hand for the left and right responses. Observer D.S. used the index and middle fingers of his right hand for the left and right responses. About 300 reactions were obtained for each of the two responses. No anticipations were recorded for Observer C.E., one was recorded for Observer D.S., and six were recorded for Observer S.V.G., three errors for each of the two possible responses.

Table 3 presents the mean and standard deviation for each stimulus-response pair. The next column of the table expresses the mean of the two responses and next to it the differences in mean reaction time for the two responses. First, note that the responses are relatively fast for a 70-dB SPL or SL signal. Second, note that there are practically no differences between the mean latencies of the two responses. Thus, either there are no large asymmetries in the audibility of either tone, or, if there are, this asymmetry is exactly counterbalanced by an opposite asymmetry in the time to execute the different responses. Because such fortuitous canceling seems unlikely, we conclude that the residual latencies are nearly equal.
When separate residuals are estimated for each response, both the SPRT and the SSR models estimate considerably more difference between the two response latencies than is evident in the simple reaction-time data. This is especially evident for the SPRT model. The best predictor of the mean of the simple reaction time is the estimated residual value for the threeparameter SPRT model, that is, when the model is forced to predict the same residual delay for the two responses. The three-parameter SSR model's predictions are nearly as good, but the average error is about $9 \mathrm{msec}$ for the SSR model and only about $3 \mathrm{msec}$ for the SPRT model.

The agreement between the simple reaction-time mean and the parameter estimated from the choice data is particularly remarkable, because similar attempts by previous investigators have produced quite different results. Laming (1979c) and Swensson (1972) have made similar calculations and found discrepancies of 50 to $100 \mathrm{msec}$. The reasons for these discrepancies are not apparent, although the methods used to estimate the residual latency, $r$, are not exactly the same. Swensson uses a linear equation expressing the observed latency as a residual, $r$, plus a term representing the accuracy of the choice. He argues that as the accuracy goes to zero, the speed of the response must be maximum, hence the value $r$. The linear estimates produce $r$ values in the 280 - to 310-msec range, whereas the fastest observed responses, with essentially chance accuracy, are about $200 \mathrm{msec}$. Laming notes that an equation true of both SSR and SPRT models is as follows:

$$
\mathbf{M}_{.1}=\mathbf{P}_{11} \mathbf{A}-\mathbf{P}_{21} \mathbf{B}
$$

and

$$
\mathbf{M}_{.2}=\mathrm{P}_{22} \mathrm{~B}-\mathrm{P}_{12} \mathrm{~A},
$$

where $\mathbf{M}_{.1}$ is the mean latency of a response to stim-

Table 3

\begin{tabular}{|c|c|c|c|c|c|c|c|c|c|c|}
\hline \multirow[b]{2}{*}{ Observer } & \multicolumn{2}{|c|}{ Response 1} & \multicolumn{2}{|c|}{ Response 2} & \multirow[b]{2}{*}{ Average } & \multirow[b]{2}{*}{ Difference } & \multirow[b]{2}{*}{ Model } & \multicolumn{2}{|c|}{ Estimated Residual } & \multirow[b]{2}{*}{ Difference } \\
\hline & Mean & 0 & Mean & $\sigma$ & & & & R1 & R2 & \\
\hline \multirow[t]{2}{*}{ D.S. } & 166 & 23 & 167 & 17 & 166 & -1 & $\begin{array}{r}\text { SPRT (4) } \\
\text { SSR (4) }\end{array}$ & $\begin{array}{l}181 \\
178\end{array}$ & $\begin{array}{l}144 \\
166\end{array}$ & $\begin{array}{l}+37 \\
+12\end{array}$ \\
\hline & & & & & & & $\begin{array}{r}\text { SPRT (3) } \\
\text { SSR (3) }\end{array}$ & \multicolumn{2}{|c|}{$\begin{array}{l}167 \\
171\end{array}$} & \\
\hline \multirow[t]{2}{*}{ C.E. } & 148 & 15 & 143 & 14 & 146 & +5 & $\begin{array}{r}\text { SPRT (4) } \\
\text { SSR (4) }\end{array}$ & $\begin{array}{l}156 \\
148\end{array}$ & $\begin{array}{l}136 \\
147\end{array}$ & $\begin{array}{r}+19 \\
+1\end{array}$ \\
\hline & & & & & & & $\begin{array}{r}\text { SPRT (3) } \\
\text { SSR (3) }\end{array}$ & \multicolumn{2}{|c|}{$\begin{array}{l}148 \\
148\end{array}$} & \\
\hline \multirow[t]{2}{*}{ S.V.G. } & 158 & 14 & 157 & 15 & 157 & +1 & $\begin{array}{r}\text { SPRT (4) } \\
\text { SSR (4) }\end{array}$ & $\begin{array}{l}154 \\
174\end{array}$ & $\begin{array}{l}168 \\
171\end{array}$ & $\begin{array}{r}-14 \\
+3\end{array}$ \\
\hline & & & & & & & $\begin{array}{r}\text { SPRT (3) } \\
\text { SSR (3) }\end{array}$ & \multicolumn{2}{|c|}{$\begin{array}{l}162 \\
173\end{array}$} & \\
\hline
\end{tabular}

Results (in Milliseconds) of Simple Reaction Time Experiment

Note-Number of parameters given in parentheses. 
ulus $\mathrm{i}$, whether right or wrong, $\mathrm{P}_{\mathrm{ij}}$ is the probability of response $i$ given stimulus $j$, and $A$ and $B$ are the border values (see Appendix). Laming adds an intercept value $r$ to each equation and estimates it by linear regression. The estimated intercept is at least $50 \mathrm{msec}$ greater than the observed value.

Using exactly this procedure on the present data produces the following values of $\mathrm{r}$ : D.S., $170 \mathrm{msec}$ $\left(R^{2}=.74\right) ;$ C.E., $150 \mathrm{msec}\left(R^{2}=.91\right)$; and S.V.G., $158 \mathrm{msec}\left(R^{2}=.94\right)$. These values are slightly different from the parameters estimated from the linear equation given in the Appendix, but their discrepancy from the measured simple reaction time is no greater than $5 \mathrm{msec}$. We are perplexed by the discrepancies found in the previous studies.

Before concluding our discussion of results, we will consider these data in terms of a mixture, rather than a random-walk, model. This general class of models is called "fast-guess models."

\section{Fast-Response Model}

Consider an observer who wishes to increase the speed of a response at the expense of accuracy. A simple way to do this is to select one response and initiate it as soon as the signal is presented, independent of which signal occurs. Such a speeded response is, in effect, a simple reaction time and can be accomplished somewhat faster than a response in which the observer waits to attempt to identify the correct stimulus alternative. Ollman (1966) has called these responses fast guesses. Obviously, on average, only half of these fast responses are correct; hence, this strategy can be pursued only infrequently, if the error rate is to be held at a low level. Typical error rates in this, and in other, choice reaction-time experiments are in the range of $5 \%$ to $10 \%$. If we assume that the speeded response is responsible for about half these errors, the effect is to reduce the latencies on error trials by about half of the difference in latency between choice and fast-guess responses. The effect on the latency of correct responses is slight, since the speeded response represents only $2.5 \%$ to $5 \%$ of all the correct responses, and would affect the mean latency by only a few milliseconds. Whatever the exact amounts, correct responses will have latencies slower than incorrect responses even if this speeded response occurs very infrequently.

The experimenter is in an awkward situation visà-vis this strategy. One can increase the penalty imposed for an incorrect response, since this should lessen the probability of a fast guess's occurring. But a reduction of the number of errors makes an estimate of the exact error probabilities more difficult. Estimates of the error probabilities are critical because they determine the boundaries of the random walk, the values $A$ and $B$. These values, in turn, appear in all the estimation equations. Thus, the experi- menter can reduce errors with larger penalties, but only at the expense of prolonging the experiment. This problem becomes further confounded when one averages over observers, since one or two observers may contribute the bulk of the errors, via speeded responses.

To evaluate the applicability of such a fast-guess model to our experiment, we compare the data of this experiment to the standard predictions made by these models. Ollman (1966) has derived the following equations:

$$
\begin{aligned}
P(1 \mid 1) L(1 \mid 1)+ & P(2 \mid 2) L(2 \mid 2) \\
& =k_{1}[P(1 \mid 1)+P(2 \mid 2)-1]+C
\end{aligned}
$$

$$
\begin{aligned}
\mathrm{P}(1 \mid 2) \mathrm{L}(1 \mid 2)+ & \mathrm{P}(2 \mid 1) \mathrm{L}(2 \mid 1) \\
& =\mathrm{k}_{2}[\mathrm{P}(1 \mid 1)+\mathrm{P}(2 \mid 2)-1]+\mathrm{C},
\end{aligned}
$$

where $P(i \mid j)$ is the probability and $L(i \mid j)$ is the latency of the $i^{\text {th }}$ response given the $j^{\text {th }}$ stimulus, $k_{1}$ and $k_{2}$ are constants, and $C$ is the mean latency of the fast guess. Figure 7 shows these kinds of plots for the three observers used in this experiment. The regression lines are also shown in the figure. The ones with positive slope are for the "correct" responses, Equation 1 , and are based on considerably more data than the lines of negative slope which represent "incorrect" responses, Equation 2. Despite the very restricted range of the independent variable, the correlation coefficients are reasonably high, the lowest being 0.96 .

The intercepts of the two equations are, according to Equations 1 and 2, estimates of the mean fastguess latencies. They are very roughly equal, except for C.E. Also plotted on the graph is the mean latency measured in the simple reaction-time experiment, the solid point at the extreme left side. In all cases, it is appreciably less than the mean estimated for a fast-guess latency. Just why this should be is something of a puzzle. Our simple reaction-time procedure is practically a simulation of the way in which a fast guess should occur. Perhaps intermixing choice and fast-guess reactions leads to somewhat slower fast guesses.

Another way to evaluate fast-guess models is to subtract Equation 2 from Equation 1, obtaining the following equation, first suggested by Yellott (1967):

$\frac{\mathrm{P}(1 \mid 1) \mathrm{L}(1 \mid 1)+\mathrm{P}(2 \mid 2) \mathrm{L}(2 \mid 2)-\mathrm{P}(1 \mid 2) \mathrm{L}(1 \mid 2)-\mathrm{P}(2 \mid 1) \mathrm{L}(2 \mid 1)}{\mathrm{P}(1 \mid 1)+\mathrm{P}(2 \mid 2)-\mathrm{P}(2 \mid 1)-\mathrm{P}(1 \mid 2)}=\mu_{s}$,

where $\mu_{s}$ is the mean of a true choice response. As Yellott points out, this is independent of $C$, the mean latency of a fast guess, which may change from condition to condition. 


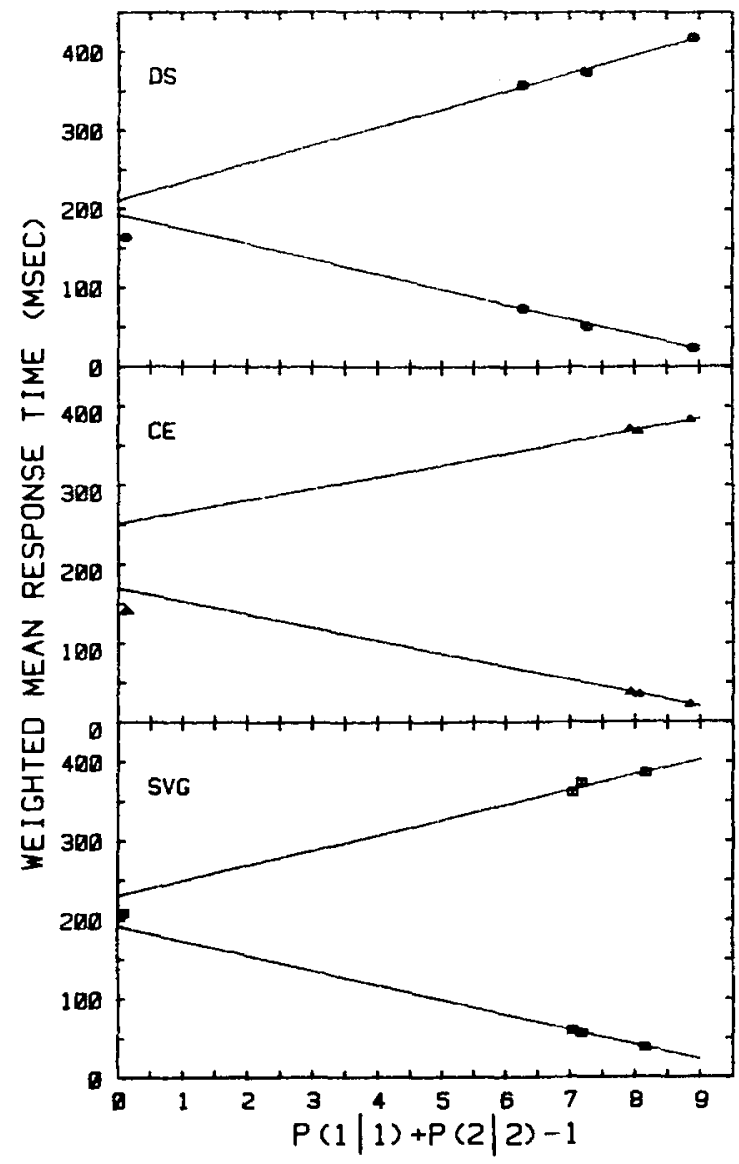

Figure 7. The data for the three conditions analyzed in accordance with Ollman's equation for the fast-guess model. The ordinate is the weighted latency of correct (or incorrect) responses plotted against the sum of the probabillties of being correct (or incorrect) minus 1. According to the theory, the relation should be linear and the Intercept should be the latency of a fast guess. The solid point plotted at the zero abseisan value is the measured Iatency of a simple reaction time.

Figure 8 shows this type of plot for our data. Again, considering the restricted range, the correlation for the individual observers is impressive. The three correlations are $.999, .972$, and .982 . A linear fit to the data yields slopes of $210 \mathrm{msec}$ (D.S.), $189 \mathrm{msec}$ (S.V.G.), and $157 \mathrm{msec}$ (C.E.), but the intercepts are not zero. The intercepts are +19 msec (D.S.), $+39 \mathrm{msec}$ (S.V.G.), and $+82 \mathrm{msec}$ (C.E.), but again, because of the amount of extrapolation, these values should not be taken too seriously. Forcing the intercept to zero and evaluating the best-fitting slope parameter, which should be an estimate of the mean latency for a true choice response yields $223 \mathrm{msec}$ (D.S.), $217 \mathrm{msec}$ (S.V.G.), and $207 \mathrm{msec}$ (C.E.). These values are happily in the same narrow range and $60 \pm 3 \mathrm{msec}$ longer than the measured means of the simple reaction times for the same observers.

The problem with these estimates is that the observed latencies should be a mixture of this true choice reaction time and the simple reaction time. Thus, the true choice estimates should represent upper bounds on the observed means, neglecting sampling error. For all of our observers, the latency for a correct response to the less frequent stimulus, in conditions in which the two signals occur with unequal probabilities, exceeds the estimated true choice reaction. For D.S., the observed values are 259 and $249 \mathrm{msec}$, for C.E., they are 229 and $229 \mathrm{msec}$, and for S.V.G., 243 and $238 \mathrm{msec}$. The observed values are, on average, $26 \mathrm{msec}$ faster than the estimated upper bound. Since these means are based on more than a thousand reactions, it seems clear that the parameters estimated from the fast-guess model are unrealistic and not accurate reflections of the observer's behavior.

\section{Summary and Conclusions}

Extensive data from a choice reaction-time experiment with three levels of a priori probability for the different stimulus alternatives have been obtained. Despite extensive previous practice, improvement in the mean reaction time was evident after the first 3,600 trials of this experiment. The mean latencies for a given response, whether correct or incorrect, are nearly equal. Two random-walk models, the sequential probability ratio test (SPRT) of Wald and Stone and the symmetric sampling representation (SSR) of Link and Heath, were compared with the data. Both models, using either three or four free parameters, could fit the mean data with an RMS error of about 1/30th of the mean value. Estimated parameter values were within reasonable ranges for

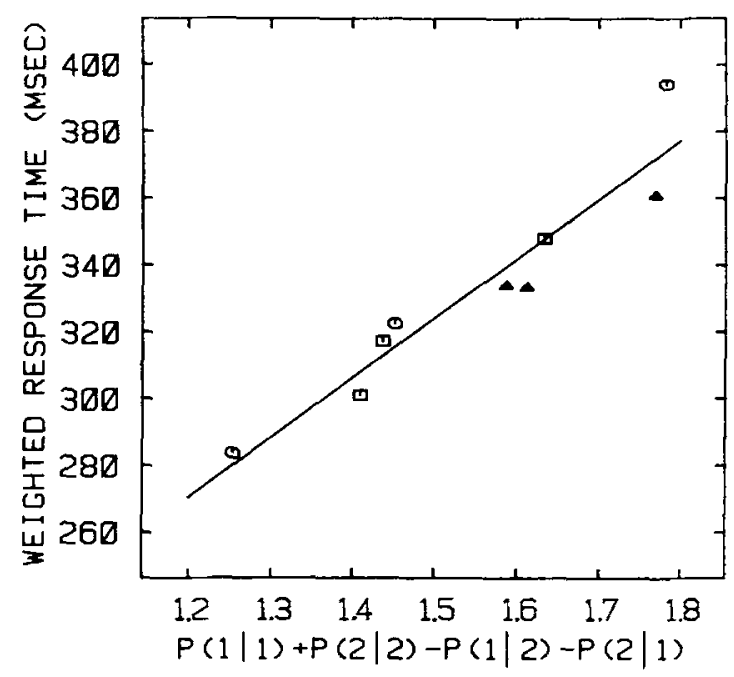

Figure 8. The data from the present experiment analyzed in accordance with Yellott's equation for the fast-guess model. The relationship should be linear with zero intercept. The slope of the line represents the mean latency of a stimulus-controlled response. The values of the estimated parameters are discussed in the text. The three observers in this experiment are coded in the following manner: D.S., circles; C.E., triangles; S.V.G., squares. 
both models. When three rather than four parameters were used (omitting a differential residual latency for each response), the basic parameters of the SSR model remained nearly the same, whereas the parameters of the SPRT model showed considerable change.

For a given response, the mean difference between correct and incorrect responses was nearly a linear function of the boundary value, either A or B, as the SSR model would predict. The intercept was not zero, however, as that theory also predicts. Little autocorrelation was evident in the data, a result different from that found by Laming. We believe our random foreperiod and heavy penalty for anticipation effectively stops presampling.

The fast-guess models predicted certain linear relations that were supported by very high correlation coefficients, despite the restricted range of the independent variable. The estimates of the mean latency for a fast guess were not the same for each of the linear relations and both estimates were larger than the mean latency for a simple reaction time. For all three observers, the residual latency estimated by the three-parameter SPRT model was almost exactly equal to the mean of the simple reaction time. The estimate, according to Yellott's equations, of the mean true choice reaction was nearly $60 \mathrm{msec}$ slower than the mean simple reaction time for all observers.

The fact that the simple reaction times were nearly the same for each response and that good fits were obtained for the three-parameter models, which ignore asymmetry between the responses, leads us to believe that the four-parameter models are inaccurate and misleading. The most serious fault of the SPRT model is that statistically one can reject the hypothesis that error and correct distributions of latency for a given response are equal. The nonzero intercept for the linear prediction of the SSR model (mean correct minus incorrect latency vs. A or B, Figure 5) is its most serious fault.

The fact that the SPRT model provides reasonable predictions of this data, and very poor predictions of some previous data, is either because more practice was given to our observers and individual data were analyzed or because there was a random foreperiod between the start of a trial and the onset of the signal, with a very heavy penalty for responses occurring before the signal was presented, or because the modality of the warning and reaction signal influences choice reaction-time behavior in ways we have, hitherto, not appreciated. Further experiments are underway to explore the nature of this discrepancy.

\section{REFERENCE NOTES}

1. Luce, R. D. Reaction times. Manuscript in preparation, 1983. 2. Noreen, D. L. Response probabilities and response time in psychophysical discrimination tasks: $A$ review of three models. Unpublished manuscript, 1976.

\section{REFERENCES}

BAmber, D. The area above the ordinal dominance graph and the area below the receiver operating characteristic graph. Journal of Mathematical Psychology, 1975, 12, 387-415.

Bradley, J. V. Distribution-free statistical tests. Englewood Cliffs, N.J: Prentice-Hall, 1968.

FITTS, P. M. Cognitive aspects of information processing: III. Set for speed vs. accuracy. Journal of Experimental Psychology, $1966,71,849-857$.

HAYs, W. L. Introduction to the theory of statistics. New York: Holt, Rinehart \& Winston, 1963.

Kendall, M. G., \& Stuart, A. Advanced theory of statistics (Vol. 2) (3rd ed.). New York: Hafner, 1960.

LAMING, D. R. J. Information theory of choice-reaction times. London: Academic Press, 1968.

Laming, D. R. J. Autocorrelation of choice reaction times. Acta Psychologica, 1979, 43, 381-412. (a)

LAmina, D. R. J. Choice reaction performance following an error. Acta Psychologica, 1979, 43, 199-224. (b)

LAming, D. R. J. A critical comparison of two random-walk models for two-choice reaction time. Acta Psychologica, 1979, 43, 431-453. (c)

Linx, S. W. The relative judgment theory of two choice response time. Journal of Mathematical Psychology, 1975, 12, 114-135.

Linx, S. W., \& HEATH, R. A. A sequential theory of psychological discrimination. Psychometrika, 1975, 40, 77-105.

Nickerson, R. S. Expectancy, waiting time, and the psychological refractory period. Acta Psychologica, 1967, 27, 23-34.

Nickerson, R. S., \& Burnhay, D. W. Response times with nonaging foreperiods. Journal of Experimental Psychology, $1969,79,452-475$.

Ollman, R. T. Fast guesses in choice reaction time. Psychonomic Science, 1966, 6, 155-156.

Picke tr, R. M. Response latency in a pattern perception situation. Acta Psychologica, 1967, 27, 160-169.

Schouten, J. R., \& Bexker, J. A. M. Reaction time and accuracy. Acta Psychologica, 1967, 27, 143-153.

Swodgrass, J. G, Foreperiod effects in simple reaction time: Anticipation or expectancy? Journal of Experimental Psychology Monograph, 1969, 79(3, Pt. 2).

Snodgrass, J. G., Luce, R. D., \& Galanter, E. Some experiments on simple and choice reaction time. Journal of Experimental Psychology, 1967, 75, 1-17.

Stone, M. Models for choice-reaction time. Psychometrika, 1960, 25, 251-260.

Swensson, R. G. The elusive tradeoff: Speed versus accuracy in visual discrimination tasks. Perception \& Psychophysics, 1972, 12, 16-32.

WAld, A. Sequential analysis. New York: Wiley, 1947.

Wolfendale, G. L. Decision times in signal detection. Acta Psychologica, 1967, 27, 154-159.

YELLTT, J. I., JR. Correction for guessing in choice reaction time. Psychonomic Science, 1967, 8, 321-322.

\section{APPENDIX}

The following are the equations used in the regression equation to estimate the theoretical quantities. The notation is $\mathbf{P}_{r s}$, where $r$ is the response and $s$ the stimulus. Similarly, $M_{12}$ refers to the mean latency of the first response given the second stimulus was presented.

For the SPRT model, there is a residual latency $r$ and two theoretical parameters $\Delta / \mu_{1}$ and $\Delta / \mu_{2}$, where $\Delta$ is a sample step per unit time and $\mu_{1}$ and $\mu_{2}$ are the means of the stimulus alternative.

$$
\mathbf{M}_{11}=\mathrm{M}_{12}=\mathrm{r}+\frac{\Delta}{\mu_{1}} \eta_{11}+\frac{\Delta}{\mu_{2}} \eta_{12}
$$




$$
\mathbf{M}_{21}=\mathbf{M}_{22}=\mathrm{r}+\frac{\Delta}{\mu_{1}} \eta_{21}+\frac{\Delta}{\mu_{2}} \eta_{22}
$$

where

$$
\begin{aligned}
& \eta_{11}=\left(A-B \frac{P_{21}}{P_{11}}\right) \frac{e^{B}}{e^{B}-e^{-A}}, \\
& \eta_{12}=\left(A-B \frac{P_{22}}{P_{12}}\right) \frac{e^{-B}}{e^{-B}-e^{A}}, \\
& \eta_{21}=\left(B-A \frac{P_{11}}{P_{21}}\right) \frac{e^{-A}}{e^{B}-e^{-A}}, \\
& \eta_{22}=\left(B-A \frac{P_{12}}{P_{22}}\right) \frac{e^{A}}{e^{-B}-e^{A}}, \\
& A=\ln \frac{P_{11}}{P_{12}} \\
& B=\ln \frac{P_{22}}{P_{21}} .
\end{aligned}
$$

For the SSR model, we follow Noreen (Note 2) and use the following equations:

$$
\mathbf{M}_{11}=\mathrm{r}+\frac{\Delta}{\mu} \eta_{\mathrm{A}}+\frac{\Delta}{\lambda \mu}\left(\eta_{\mathrm{A}}-\mathbf{A}\right),
$$

$$
\mathbf{M}_{12}=\mathrm{r}+\frac{\Delta}{\mu}\left(\eta_{\mathrm{A}}-\mathrm{A}\right)+\frac{\Delta}{\lambda \mu} \cdot \eta_{\mathrm{A}},
$$

$$
\mathbf{M}_{21}=\mathrm{r}+\frac{\Delta}{\mu}\left(\eta_{\mathrm{B}}-\mathrm{B}\right)+\frac{\Delta}{\lambda \mu} \eta_{\mathrm{B}},
$$

$$
\mathbf{M}_{22}=\mathbf{r}+\frac{\Delta}{\mu} \eta_{\mathrm{B}}+\frac{\Delta}{\lambda \mu}\left(\eta_{\mathrm{B}}-\mathrm{B}\right),
$$

where

$$
\begin{gathered}
\eta_{A}=\frac{P_{22}}{P_{11}+P_{22}-1}\left[A P_{11}-\mathbf{B}\left(1-P_{11}\right)\right], \\
\eta_{B}=\frac{P_{11}}{P_{11}+P_{22}-1}\left[\mathbf{P P}_{22}-\mathbf{A}\left(1-\mathbf{P}_{22}\right)\right], \\
A=\ln \frac{P_{11}}{\mathbf{P}_{12}}, \\
B=\ln \frac{\mathbf{P}_{22}}{\mathbf{P}_{21}} .
\end{gathered}
$$

For the four-parameter model, we added a fourth parameter response, which was weighted +1 when the first response occurred and -1 when the second response occurred. 